\title{
Mercaptobenzothiazole or the mercapto-mix: which should be in the standard series?
}

\author{
Thomas L. Diepgen ${ }^{1}$, Derk P. Bruynzeel ${ }^{2}$, Klaus E. Andersen ${ }^{3}$, Francisco M. Brandão ${ }^{4}$, Magnus Bruze ${ }^{5}$, \\ Margarida Gonçalo $^{6}$, An Goossens ${ }^{7}$, Arto Lahti ${ }^{8}$, Vera Mahler ${ }^{9}$, Torkil Menné ${ }^{10}$, Ian R. White ${ }^{11}$ and John \\ D. Wilkinson ${ }^{12}$ on behalf of the European Environmental Contact Dermatitis Research Group (EECDRG) \\ ${ }^{1}$ Departments of (Occupational) Dermatology, University Hospital, Heidelberg, Germany, ${ }^{2}$ Departments of \\ (Occupational) Dermatology, VU University Medical Centre, Amsterdam, the Netherlands, ${ }^{3}$ Departments of \\ (Occupational) Dermatology, Odense University Hospital, University of Southern Denmark, ${ }^{4}$ Departments of \\ (Occupational) Dermatology, Hospital Garcia de Orta, Almada, Portugal, ${ }^{5}$ Departments of (Occupational) \\ Dermatology, University Hospital Malmo, Sweden, ${ }^{6}$ Departments of (Occupational) Dermatology, University \\ Hospital Coimbra, Portugal, ${ }^{7}$ Departments of (Occupational) Dermatology, University Hospital KU Leuven, Belgium, \\ ${ }^{8}$ Departments of (Occupational) Dermatology, University of Oulu, Finland, ${ }^{9}$ Departments of (Occupational) \\ Dermatology, University Hospital, Erlangen, Germany, ${ }^{10}$ Gentofte Hospital, Copenhagen, Denmark, ${ }^{11}$ Departments \\ of (Occupational) Dermatology, St. Thomas' Hospital, London, and ${ }^{12}$ Departments of (Occupational) Dermatology, \\ Amersham Hospital, Bucks, UK
}

\begin{abstract}
Mercaptobenzothiazole (MBT) compounds are well known contact allergens. To detect rubber allergic patients we use both MBT ( $2 \%$ in petrolatum) and a mercapto-mix with 4 constituents of $0.5 \%$ each in our standard series. In this article the EECDRG presents data of in total 32475 consecutive tested patients attending the respective contact dermatitis clinics from 11 centres in Europe to determine if the mix and MBT detected the same allergic patients. We found 327 patients positive to the mix or MBT, or to both. 261 were positive to the mix and 254 to MBT. MBT was negative in 73 patients who were positive to the mix. If the mix had not been in the standard series, on average $22 \%$ of patients allergic to a mercapto-compound would have been missed, for MBT this would have been on average $20 \%$. All clinics would have missed a significant number of positive reactions if both compounds had not been tested. We conclude, that both the mercapto mix and MBT are required in the standard series.
\end{abstract}

Key words: contact dermatitis; delayed-type allergy; European standard series; mercapto-mix; mercaptobenzothiazole. (c) 2006 The Authors. Journal compilation (c) 2006 Blackwell Munksgaard.

Accepted for publication 23 February 2006

Mercaptobenzothiazole compounds are wellknown contact allergens. They can be found in rubber products as accelerators but they are also used as antirust agents and fungicides, e.g. in metal working fluids and agricultural pesticides. Mercaptobenzothiazole (MBT) 2\% in petrolatum was part of the first 20 standard patch test allergens proposed by the ICDRG (International Contact Dermatitis Research Group) in $1968(1,2)$. In the 1970s MBT was replaced by a mix $(3,4)$ which consisted of 4 chemicals each in a concentration of $0.25 \%$ : 2-mercaptobenzothiazole (MBT), N-cyclohexyl-2-benzothiazyl sulfenamide, 2,2'dibenzothiazyl disulphide and 2(4-morpholinyl)mercaptobenzothiazole. Some years later the concentration of each chemical was raised to 0.5\% (5). In 1979 the North American Contact Dermatitis Group advised a mercapto-mix without MBT, the concentrations of the 3 remaining 3 constituents were $0.333 \%$. MBT itself was tested in a concentration of $1 \%$ in petrolatum; investigations were suggestive that otherwise mercaptocompounds allergic patients were missed (6). In 1988 the ICDRG and the EECDRG recommended adding MBT 2\% to the standard series to avoid false negative reactions (7). Hansson \& Agrup (1993) (8) reported on the instability of the mercapto-mix substances and as MBT was the stable end-product they proposed using MBT as the single chemical for patch testing for mercaptobenzothiazole/derivatives sensitivity. Shortly afterwards Geier \& Gefeller (1995) (9), using data obtained from the Information Network of Departments of Dermatology (IVDK) in Germany, that the mix could be replaced by MBT.

15 years later we are still using MBT in combination with a mix in our standard series. 
Even the test concentrations still differ between the American and the European series. The EECDRG recommends MBT 2\% and a mix with 4 constituents of $0.5 \%$ each, the NACDG advises MBT $1 \%$ in combination with the mix with 3 chemicals each $0.333 \%$. For practical reasons, the ICDRG follows the advice of the NACDG (10).

The EECDRG reviewed their results of the European standard series for the years 1996-2000 (11). The results of patch testing about 26000 patients raised again the question of having both MBT and the mercapto-mix in the standard series as both test preparations were positive in I $\%$ of the tested population. The data from clinics of the EECDRG were used to determine if the mix and MBT detected the same allergic patients.

\section{Patients and Methods}

The data was retrospectively compiled for the period 1996 through 2000 from 11 centres in Europe. Included were consecutive tested patients attending the respective contact dermatitis clinics, in total 32475 patients.

Patch testing was performed according to the ICDRG guidelines with the European Standard series (e.g. MBT 2\% and mercapto-mix 2\%). The results were based on the final evaluation of the patch testing in each clinic, usually the day 3 or 4 reading. Finn chambers ${ }^{\mathbb{B}}$ were used in 8 and Van der Bend Square Chambers ${ }^{\mathbb{R}}$ in 2 centres. One centre used the TRUE Test ${ }^{\mathbb{R}}$ system.

\section{Results}

The data of each clinic is given in Table 1: the total number of patch-tested patients and those who were positive for mercapto-compounds, either the mix or MBT and the number of patient not reacting to one of these two compounds. The quotient of these figures gives the percentage of patients which would not have been identified if the mix or MBT was not included in the European Standard Series. If the mix had not been in the standard series, on average $22 \%$ of the patient allergic to a mercaptocompound would have been missed. The variation between the involved clinics ranged between $0 \%$ and $34 \%$. This is due to the small numbers of positive reaction in the separate clinics. For MBT this would have been on average $20 \%$ if this substance had been omitted (range $0-32 \%$ ).

The combined figures of the 11 clinics are shown in Table 2. If all figures are added together we have 327 patients positive to the mix or MBT, or to both. 261 were positive to the mix and 254 to MBT. MBT was negative in 73 patients who were positive to the mix.

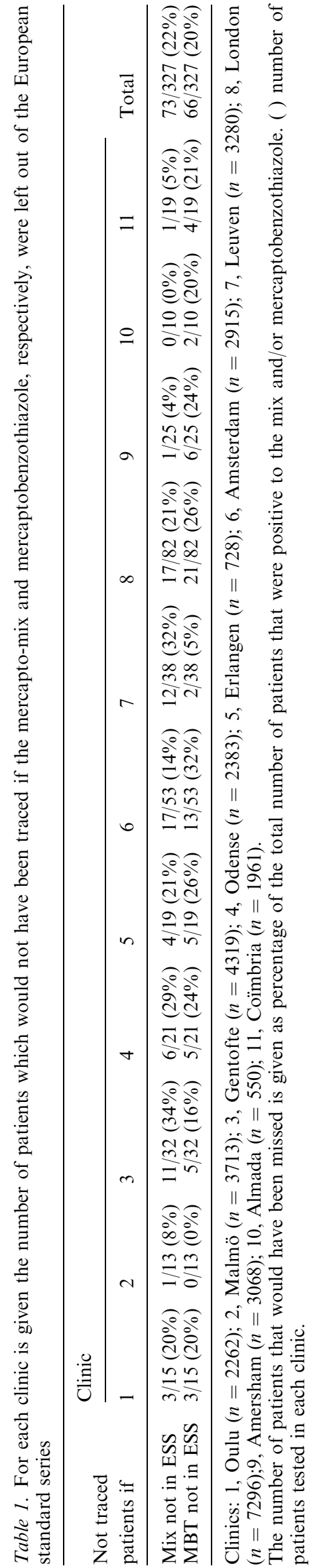


Table 2. Number of patients with a positive or negative reaction for mercaptobenzothiazole and the mercapto-mix in the European standard series

\begin{tabular}{lrlr}
\hline & MBT positive & MBT negative & Total \\
\hline Mercapto-mix pos. & 188 & 73 & 261 \\
Mercapto-mix neg. & 66 & - & 66 \\
Total & 254 & 73 & 327 \\
\hline
\end{tabular}

The results are compiled from 11 clinics throughout Europe; total number of patients tested were 32475 , out of which 327 patients were sensitized for at least one of the two Mercaptocompounds.

Practically all clinics would have missed a significant number of positive reactions if both compounds had not been tested. There is no important difference between the clinic $\left(\mathrm{n}^{\circ} 4\right)$ using the TRUE Test ${ }^{\mathbb{R}}$ system and the other clinics.

\section{Discussion}

Our study shows clearly, in a large group of patients (327 mercapto-compound positive patients), that both the mercapto-mix and MBT are required in the standard series. This confirms other studies which also indicate that a mix in combination with MBT is necessary to detect all your mercapto-sensitive patients $(12,13)$. Missing on an average of about $20 \%$ and $22 \%$, respectively, of sensitized patients is unacceptable.

Based on cross-reactivity studies and a study on the stability of the compounds of the mix it would be sufficient to test with MBT only $(8,14$, 15). Several clinical studies suggested that this may be correct and that MBT alone would suffice $(9,16)$. However, our study clearly demonstrates that a large number of patients would be needed to be able to confirm such a conclusion.

Adding only $0.2 \%$ of patients with contact allergy by putting an additional allergen to the standard series does not sound reasonable (17). However, it detects in this case an important extra amount of rubber allergic patients. Of course it would be better to have only one allergen or mix to detect 'all' mercapto-allergic patients. But, there is no alternative so far.

The test concentration of MBT, 1 or $2 \%$, the composition of the mercapto-mix and the concentration of each compound in the mix have been changed several times since the introduction in 1968. Extensive research into the best solution is lacking. It would be interesting to have data comparing the American mercapto-mix with 3 components (not including MBT) and the mix with 4 components (including MBT) used in Europe. The combination of 3 components in combination with MBT as a separate test material sounds more logical then the present European approach.

For the moment it is advisable to continue with European Standard Series as it is: MBT and the mercapto-mix (4 components) both $2 \%$ in petrolatum (18).

\section{References}

1. ICDRG. Patch test allergens (Editorial). Contact Dermatitis Newsletter 1968: 38.

2. ICDRG. Patch test allergens (Editorial). Contact Dermatitis Newsletter 1969: 82-84.

3. Cronin E. Clinical prediction of patch testing results. Trans St Johns Hosp Dermatol Soc 1972: 58: 153-162.

4. Fregert S, Bandmann H J. Patch Testing. Berlin Heidelberg, New York, Springer-Verlag, 1975: 6-7.

5. Malten K E, Nater J P, van Ketel W G. Patch Testing Guidelines. Nijmegen, Dekker \& van de Vegt, 1976: 96-98.

6. Mitchel J C. Patch testing with mixes. Contact Dermatitis 1981: 7: 98-104.

7. Andersen K E, Burrows D, Cronin E, Dooms-Goossens A, Rycroft R J G, White I R. Recommended changes to standard series. Contact Dermatitis 1988: 389-390.

8. Hansson C, Agrup G. Stability of the mercaptobenzothiazole compounds. Contact Dermatitis 1993: 28: 29-34.

9. Geier J, Gefeller O. Sensitivity of patch tests with rubber mixes: results of the information network of departments of dermatology from 1990 to 1993. Am J Contact Dermat 1995: 6: 143-149.

10. Lachapelle J-M, Maibach H I. Patch Testing and Prick Testing. Berlin, Springer-Verlag, 2003: 73-79.

11. Bruynzeel D P, Diepgen T L, Andersen K E et al. Monitoring the European Standard Series in 10 centres: 1996-2000. Contact Dermatitis 2005: 53: 146-149.

12. Lynde C W, Mitchell R M, Adams R M, Maibach H I, Schorr W J, Storrs F J, Taylor J. Patch testing with mercaptobenzothiazole and mercapto-mixes. Contact Dermatitis 1982: 8: 273-274.

13. Lamintausta K, Kalimo K. Sensitivity to rubber. Dermatosen 1985: 33: 204-208.

14. Fregert S. Cross-sensitivity pattern of 2-Mercaptobenzothiazole (MBT). Acta Derm Venereol 1969: 49: 45-48.

15. Wang X, Suskind R R. Comparative studies of the sensitization potential of morpholine, 2-mercaptobenzothiazole and 2 of their derivates in guinea pigs. Contact Dermatitis 1988: 19: 11-15.

16. Knudsen B B, Hametner L, Seycek O, Heesch A, Kak H-U, Peters K-P. Allergologically relevant rubber accelerators in single-use medical gloves. Contact Dermatitis 2000: 43: 9-15.

17. Bruze M, Conde-Salazar L, Goossens A, Kanerva L, White I R. Thoughts on sensitizers in a standard patch test series. The European Society of Contact Dermatitis. Contact Dermatitis 1999: 41: 241-250.

18. Isaksson $\mathrm{M}$, Brandao $\mathrm{F} \mathrm{M}$, Bruze $\mathrm{M}$, Goossens $\mathrm{A}$. Recommendation to include budesonide and tixocortol pivalate in the European standard series. Contact Dermatitis 2000: 43: 41-42.

Address:

T. L. Diepgen

Department of Social Medicine

Occupational \& Environmental Dermatology

University Hospital Heidelberg

Thibaut Str. 3

69115 Heidelberg

Germany

Tel: +496221568751

Fax: + 496221565019

e-mail: thomas_diepgen@med.uni-heidelberg.de 